\title{
The Hilbert Space of Double Fourier Coefficients for an Abstract Wiener Space
}

\author{
Jeong-Gyoo Kim
}

School of Games, Hongik University, Sejong 30016, Korea; jgkim@hongik.ac.kr or jgkimath@yonsei.ac.kr

\begin{abstract}
Fourier series is a well-established subject and widely applied in various fields. However, there is much less work on double Fourier coefficients in relation to spaces of general double sequences. We understand the space of double Fourier coefficients as an abstract space of sequences and examine relationships to spaces of general double sequences: $p$-power summable sequences for $p=1,2$, and the Hilbert space of double sequences. Using uniform convergence in the sense of a Cesàro mean, we verify the inclusion relationships between the four spaces of double sequences; they are nested as proper subsets. The completions of two spaces of them are found to be identical and equal to the largest one. We prove that the two-parameter Wiener space is isomorphic to the space of Cesàro means associated with double Fourier coefficients. Furthermore, we establish that the Hilbert space of double sequence is an abstract Wiener space. We think that the relationships of sequence spaces verified at an intermediate stage in this paper will provide a basis for the structures of those spaces and expect to be developed further as in the spaces of single-indexed sequences.
\end{abstract}

Keywords: double Fourier series; Cesàro sum of double Fourier coefficients; $\ell^{p}$ space of double sequences; Hilbert space of double sequences; abstract Wiener space; two-parameter Wiener space

MSC: 42A16; 42B05; 46A45; 46B45; 46C99

Citation: Kim, J.-G. The Hilbert Space of Double Fourier Coefficients for an Abstract Wiener Space. Mathematics 2021, 9, 389. https://doi.org/ $10.3390 /$ math 9040389

Received: 17 January 2021

Accepted: 10 February 2021

Published: 15 February 2021

Publisher's Note: MDPI stays neutral with regard to jurisdictional claims in published maps and institutional affiliations.

\section{Introduction}

Fourier series is one of the well-established subjects in mathematics and is widely applied in many fields of science and engineering. In addition to this utility, a space consisting of Fourier coefficients of a one-variable function has been studied well in relation to sequence spaces. However, there is much less work on double Fourier coefficients in relation to spaces of double sequences.

In this paper, we are interested in the space of sequences composed of double Fourier coefficients, whose member is from a Fourier series of a two-variable function, often called a double Fourier series. We explore the space in relation to other familiar spaces of double sequences. In our study four spaces are discussed: namely, $p$-power summable sequences for $p=1,2$, the Hilbert space of double sequences and the double sequences space of Cesàro means (also called Cesàro summations) associated with double Fourier series. We verified inclusion relationships and more properties of these four spaces. Though it is an intermediate stage to achieve our ultimate goal to an abstract Wiener space, we think that our results at this point are significant and can provide a basis for structures of those spaces. We expect that our results can be developed further as in the spaces of single-indexed sequences.

We also derive an isomorphic relationship mapped from the space of square-integrable functions to the space of 2-power summable sequences; the isomorphism maps the twoparameter Wiener space to the space of double Fourier coefficients. This enables us to achieve an abstract Wiener space of the Hilbert space of double sequences.

The representation of double Fourier series and detailed calculation to derive its formula can be found in [1]; uniform convergence of continuously differentiable functions 
of two variables is also explained therein. We refer to Wu's work [2] for the concept of a Ceràso mean and Fejér theorem, as the theorem [3] is written in French.

For an abstract Wiener space we mainly refer to the work in [4] by Kuo. Though the concept of an abstract Wiener space is known to first appear in [5] by Gross, we cite necessary definitions and properties from the work in [4]. The background provided in this paper is sufficient to understand the second half of this paper without further references on an abstract Wiener space. Other mathematical terms used there are those that can be easily found in books on functional analysis.

There are very few closely related works; the space of Fourier coefficients relating to an analog of Wiener space is studied in [6], whereas the authors of [7] discuss them in relation to an abstract Wiener space. Both are for the spaces of single-indexed sequences. To the best of the author's knowledge, no work on spaces of double sequence relating to an abstract Wiener space.

In the next section, we introduce double Fourier expansion and Fejér theorem on single-indexed sequences for background knowledge. In Section 3, we discuss four spaces of double sequences including double Fourier coefficients and verify their inclusion relationships. Section 4 provides an isometric isomorphism between the space of double Fourier coefficients and the two-parameter Wiener space. We establish an abstract Wiener space of the Hilbert space of double sequences to the space of square summable sequence in Section 5. Finally, conclusions and discussions are given in the last section.

\section{Preliminaries}

\subsection{Fourier Series}

We introduce preliminary concepts of Fourier series and spaces of sequences necessary for our main theorems in later sections.

Notations. A sequence is denoted by $\left\{a_{m, n}\right\}_{m, n=0}^{\infty}$ or simply $\left\{a_{m, n}\right\}$.

Definition 1. Let $S$ and $T$ be real numbers. A function $f: \mathbb{R} \times \mathbb{R} \rightarrow \mathbb{R}$ is said to be $(S, T)$-periodic in variables $s$ and t provided that $f(s+S, t+T)=f(s, t)$ holds for all real numbers $s$ and $t$.

The derivation procedures of double Fourier series are explained in detail in [1], Chapter 6, pp. 141-145. For the simplicity of constants in front of integrals we follow the notations in [8].

Definition 2 (Trigonometric form [8]). Let a function $f: \mathbb{R} \times \mathbb{R} \rightarrow \mathbb{R}$ be continuous on $[-S, S] \times[-T, T]$ and periodic with period $(2 S, 2 T)$. Then, the following series is called the double Fourier series of $f$ :

$$
\begin{aligned}
f(x, y) & =\sum_{m, n=0}^{\infty}\left\{A_{m, n}(f) \cos \frac{m \pi x}{S} \cos \frac{n \pi y}{T}+B_{m, n}(f) \sin \frac{m \pi x}{S} \cos \frac{n \pi y}{T}\right. \\
& \left.+C_{m, n}(f) \cos \frac{m \pi x}{S} \sin \frac{n \pi y}{T}+D_{m, n}(f) \sin \frac{m \pi x}{S} \sin \frac{n \pi y}{T}\right\},
\end{aligned}
$$

where

$$
\begin{aligned}
A_{m, n}(f) & =\frac{1}{S T} \int_{-S}^{S} \int_{-T}^{T} f(x, y) \cos \frac{m \pi x}{S} \cos \frac{n \pi y}{T} d x d y, \\
B_{m, n}(f) & =\frac{1}{S T} \int_{-S}^{S} \int_{-T}^{T} f(x, y) \sin \frac{m \pi x}{S} \cos \frac{n \pi y}{T} d x d y, \\
C_{m, n}(f) & =\frac{1}{S T} \int_{-S}^{S} \int_{-T}^{T} f(x, y) \cos \frac{m \pi x}{S} \sin \frac{n \pi y}{T} d x d y, \\
D_{m, n}(f) & =\frac{1}{S T} \int_{-S}^{S} \int_{-T}^{T} f(x, y) \sin \frac{m \pi x}{S} \sin \frac{n \pi y}{T} d x d y .
\end{aligned}
$$


Remark 1 (Parseval's formula [1]). For the notations above, we have

$$
\frac{1}{S T} \int_{-S}^{S} \int_{-T}^{T} f(x, y)^{2} d x d y=\sum_{m, n=0}^{\infty}\left\{A_{m, n}(f)^{2}+B_{m, n}(f)^{2}+C_{m, n}(f)^{2}+D_{m, n}(f)^{2}\right\} .
$$

We will need the property that the mean sequence of partial sums of double Fourier series converges uniformly in later sections. Such property for one-variable functions is known to be Fejér theorem [3] which is written in French and German. We refer to its English explanation in Wu's work of 2004 [2]. As no literature of Fejér theorem for double series has been found yet, we need to provide its proof. Our proof for a double Fourier series will be similar to that of Fejér. Therefore, we introduce Fejér's theorem here and we added details to the proof of [2] because they will also be our proof for a double Fourier series presented at the end of this section.

Proposition 1 (Fejér in [2]). Let $f$ be continuous periodic function on $[-\pi, \pi]$ with period $2 \pi$. Let $\hat{f}(k):=\frac{1}{2 \pi} \int_{-\pi}^{\pi} f(x) e^{-i k x} d x$. The Fejér's kernel $F_{n}(x)$ is defined and its properties are as follows:

$$
D_{k}(x):=\sum_{m=-k}^{k} e^{-i m x}=\left\{\begin{array}{ll}
\frac{\sin \left(\frac{(2 k+1) x}{2}\right)}{\sin (x / 2)} & (x \neq 0, \pm \pi) \\
2 k+1 & (x=0 \text { or } \pm \pi)
\end{array} \text { and } D_{k}(x) \text { is continuous at } 0 .\right.
$$

$F_{n}(x):=\frac{1}{n+1} \sum_{k=0}^{n} D_{k}(x)$ for $x \neq 0$, and $F_{n}(0)=n+1$. Then $F_{n}(x)=\frac{\sin ^{2} \frac{(n+1) x}{2}}{(n+1) \sin ^{2} \frac{x}{2}}$ and is continuous.

For a partial sum $s_{n}(x):=\sum_{k=-n}^{n} \hat{f}(k) e^{i k x}$ and its arithmetic mean

$\sigma_{n}(x):=\frac{1}{n+1} \sum_{k=0}^{n} s_{k}(x)=\int_{-\pi}^{\pi} f(x-t) F_{n}(t) d t$, we have the following properties.

$s_{n}(x)=\frac{1}{2 \pi} \int_{-\pi}^{\pi} f(x-t) D_{n}(t) d t$.

$\frac{1}{2 \pi} \int_{-\pi}^{\pi} F_{n}(x) d x=1$.

(5) For each $\delta>0, \lim _{n \rightarrow \infty} \int_{\delta \leq|x| \leq \pi} F_{n}(x) d x=0$, and

(6) $\left\{\sigma_{n}(x)\right\}$ converges to $f$ uniformly.

In the last statement, the limit of $\left\{\sigma_{n}(x)\right\}$ is often called a Cesàro mean or Cesàro summation.

Proof. Let $w:=e^{i x}$ for a non-zero real number $x$.

$$
\begin{aligned}
& D_{k}(x)=\sum_{n=-k}^{k} w^{k}=\sum_{n=-k}^{-1} w^{k}+\sum_{n=0}^{k} w^{k}=\frac{w^{-k}-1}{1-w}+\frac{1-w^{k+1}-1}{1-w}=\frac{w^{k+1}-w^{-k}}{w-1} \frac{w^{-1 / 2}}{w^{-1 / 2}} \\
& \quad=\frac{\left\{w^{k+1 / 2}-w^{-(k+1 / 2)}\right\} / 2}{\left\{w^{1 / 2}-w^{-1 / 2}\right\} / 2}=\frac{\sin ((2 k+1) x / 2)}{\sin (x / 2)} \text { by DeMoivre's theorem. As } \\
& \lim _{x \rightarrow 0} D_{k}(x)=2 k+1, D_{k}(x) \text { is continuous at } 0 .
\end{aligned}
$$

(2) Using (1) and trigonometric identities,

$$
\begin{aligned}
& F_{n}(x)=\frac{1}{n+1} \sum_{k=0}^{n} D_{k}(x)=\frac{\sum_{k=0}^{n} \sin \frac{x}{2} \sin \frac{(2 k+1) x}{2}}{(n+1) \sin ^{2} \frac{x}{2}}=\frac{\sum_{k=0}^{n}\left\{\cos \left(\frac{x}{2}-\frac{(2 k+1) x}{2}\right)-\cos \left(\frac{x}{2}+\frac{(2 k+1) x}{2}\right)\right\}}{2(n+1) \sin ^{2} \frac{x}{2}} \\
& =\frac{(\cos 0-\cos x)+(\cos (-x)-\cos 2 x)+\cdots+(\cos (-n x)-\cos (n+1) x)}{2(n+1) \sin ^{2} \frac{x}{2}}=\frac{1-\cos (n+1) x}{2(n+1) \sin ^{2} \frac{x}{2}}=\frac{\sin ^{2} \frac{(n+1) x}{2}}{(n+1) \sin ^{2} \frac{x}{2}} . \\
& \text { As } \lim _{x \rightarrow 0} F_{n}(x)=n+1 \text { and } \\
& F_{n}(0)=\frac{1}{n+1} \sum_{k=0}^{n}(2 k+1)=\frac{1}{n+1}[n(n+1)+(n+1)]=n+1, F_{n}(x) \text { is continuous. }
\end{aligned}
$$

(3) By the substitution theorem for integration, we can easily show the equality in (3):

$$
s_{n}(x)=\sum_{k=-n}^{n} \hat{f}(k) e^{i k x}=\frac{1}{2 \pi} \sum_{k=-n}^{n} \int_{-\pi}^{\pi} f(u) e^{i k(x-u)} d u=\frac{1}{2 \pi} \int_{-\pi}^{\pi} f(x-t) D_{n}(t) d t .
$$

$$
\begin{aligned}
& \text { As } \frac{1}{2 \pi} \int_{-\pi}^{\pi} e^{-i m x} d x=\left\{\begin{array}{cc}
0 & (m \neq 0) \\
1 & (m=0)
\end{array},\right. \\
& \frac{1}{2 \pi} \int_{-\pi}^{\pi} F_{n}(x) d x=\frac{1}{n+1} \sum_{k=0}^{n} \sum_{m=-k}^{k} \frac{1}{2 \pi} \int_{-\pi}^{\pi} e^{-i m x} d x=\frac{n+1}{n+1}=1 .
\end{aligned}
$$


(5) Let $0<\delta<\pi$ be given.

$0 \leq \int_{\delta \leq|x| \leq \pi} F_{n}(x) d x=\int_{\delta \leq|x| \leq \pi} \frac{\sin ^{2} \frac{(n+1) x}{2}}{(n+1) \sin ^{2} \frac{x}{2}} d x \leq \frac{1}{n+1} \int_{-\pi}^{\pi} \frac{1}{\sin ^{2} \frac{\delta}{2}} d x \rightarrow 0$ as $n$ goes to $\infty$.

(6) Let $\epsilon>0$ be given. As $f$ is uniformly continuous, there is a $\delta>0$ such that $|x-y|<\delta$ implies $|f(x)-f(y)|<\epsilon$. Then,

$$
\begin{aligned}
& \left|\sigma_{n}(x)-f(x)\right|<\frac{1}{2 \pi} \int_{-\pi}^{\pi}|f(t-x)-f(x)| F_{n}(t) d t \\
& =\frac{1}{2 \pi} \int_{|t|<\delta}|f(t-x)-f(x)| F_{n}(t) d t+\frac{1}{2 \pi} \int_{\delta \leq|t| \leq \pi}|f(t-x)-f(x)| F_{n}(t) d t \\
& \leq \epsilon \frac{1}{2 \pi} \int_{-\pi}^{\pi} F_{n}(t) d t+\left(\max _{|t| \leq \pi}|f(t)|\right) \frac{1}{2 \pi} \int_{\delta \leq|t| \leq \pi} F_{n}(t) d t \leq \epsilon \text { as } n \rightarrow \infty .
\end{aligned}
$$

In the next theorem, we provide the convergence of the sequence of arithmetic means of partial Fourier sums for a periodic function $f(x, y)$, which is necessary for our theorems in Section 4. The theorem can be called Fejér theorem for two-variable functions.

Theorem 1. Let $f$ be a continuous function defined on $[-\pi, \pi] \times[-\pi, \pi]$ and periodic with period $(2 \pi, 2 \pi)$. Let

$$
\begin{aligned}
& \hat{f}(k, l):=\frac{1}{(2 \pi)^{2}} \int_{-\pi}^{\pi} \int_{-\pi}^{\pi} f(x, y) e^{-i k x} e^{-i l y} d x d y ; \\
& D_{k, l}(x, y):=\sum_{m=-k}^{k} \sum_{n=-l}^{l} e^{-i m x} e^{-i l y} ; \\
& F_{m, n}(x, y):=\frac{1}{(m+1)(n+1)} \frac{\sin ^{2} \frac{(n+1) x}{2}}{\sin ^{2} \frac{x}{2}} \frac{\sin ^{2} \frac{(n+1) y}{2}}{\sin ^{2} \frac{y}{2}}=\frac{1}{(m+1)(n+1)} \sum_{k=0}^{n} D_{m, n}(x, y) ; \\
& s_{m, n}(x, y):=\sum_{k=-m}^{m} \sum_{l=-n}^{n} \hat{f}(k, l) e^{i k x} e^{i l y}=\frac{1}{(2 \pi)^{2}} \int_{-\pi}^{\pi} \int_{-\pi}^{\pi} f(t-x, p-y) D_{m, n}(t, p) d t d p ; \\
& \sigma_{m, n}(x, y):=\frac{1}{(m+1)(n+1)} \sum_{l=0}^{m} \sum_{k=0}^{n} s_{k, l}(x, y)=\int_{-\pi}^{\pi} \int_{-\pi}^{\pi} f(x-t, y-p) F_{m, n}(t, p) d t d p .
\end{aligned}
$$

Then,

$$
\begin{aligned}
& \text { (a) } \quad \frac{1}{(2 \pi)^{2}} \int_{-\pi}^{\pi} \int_{-\pi}^{\pi} F_{m, n}(x, y) d x d y=1 ; \\
& \text { (b) } \text { for each } \delta>0, \lim _{m, n \rightarrow \infty} \int_{\delta \leq \sqrt{x^{2}+y^{2}} \leq \pi} F_{m, n}(x, y) d x d y=0 \text {; } \\
& \text { (c) }\left\{\sigma_{m, n}(x, y)\right\} \text { converges to } f \text { uniformly. }
\end{aligned}
$$

Proof. Let $f(x, y)$ be a two-variable function defined on $[-\pi, \pi] \times[-\pi, \pi]$. We deal with it as a single variable function and use the method of Fejér. First, we fix $y$ in $f(x, y)$, then our function $f(x, y)$ can be regraded as the $f(x)$ in Proposition 1 and $D_{m, n}, F_{m, n}$, and $s_{m, n}$ are represented by single summations. Next, we consider $y$ as a variable too, and obtain corresponding double summations to them. With the double summations, we can proceed in a similar manner to Proposition 1 to obtain the statements (a), (b), and (c).

We will also call the limit of $\left\{\sigma_{m, n}(x, y)\right\}$ a Cesàro mean or Cesàro summation in this paper as in single-indexed sequences.

Remark 2. Using the same procedure as in Theorem 1, we can prove Fejér's theorem for nvariable functions.

\subsection{Abstract Wiener Space}

We give frequently used notations and definitions which can be easily found in functional analysis books. More definitions for an abstract Wiener space will be provided in the last section where they are necessary. 


\section{Notations.}

- $\quad R=[0, S] \times[0, T]$ in $\mathbb{R} \times \mathbb{R}$.

- $\quad L^{2}(X)$ is the set of all functions $f$ for which $\|f\|_{2}=\left(\int_{X}|f|^{2} d \mu\right)^{1 / 2}$, where $\mu$ is a positive measure and $X$ is a metric space.

- $\quad C(R)$ denotes the space of all real-valued continuous functions defined on $R$.

- $C_{0}(R)=\{x \mid x(\cdot, \cdot): R \rightarrow \mathbb{R}, x$ is continuous on $R$, and $x(0, \cdot)=x(\cdot, 0)=0\}$.

- $\quad H$ be a real separable Hilbert space with norm $\|\cdot\|=\sqrt{\langle\cdot, \cdot\rangle}$.

- $\quad P$ is an orthogonal projection on $H$.

- $\mathcal{F}$ is the partially ordered set of orthogonal projections $P$ of $H(P>Q$ means $P(H) \supset$ $Q(H)$ for $P, Q \in \mathcal{F})$.

\section{Inclusion of Spaces of Double Sequences: $\ell^{1}, \ell^{2}, \mathcal{H}$, and $\mathcal{U}$}

In this section, we introduce spaces of double sequences of real numbers and discuss their relationships of inclusion, namely, $\mathcal{H}, \ell^{1}, \mathcal{U}$, and $\ell^{2}$. Among them $\mathcal{U}$ is defined in association with double Fourier series. Before defining the spaces, we discuss double Fourier coefficients introduced in Section 2, especially, of a symmetric function, and a sequence consisting of its double Fourier coefficients.

Definition 3. Let $R=[0, S] \times[0, T]$ and $F(R)$ be the space of two-variable functions on $R$. For $x$ in $F(R)$, we define $\tilde{x}$ as a periodic function having period $(2 S, 2 T)$ such that

$$
\begin{aligned}
& \tilde{x}(s, t)=x(s, t) \text { for }(s, t) \text { in } R, \\
& \tilde{x}(s, t)=x(-s, t) \text { for }-S \leq s<0,0 \leq t<T, \\
& \tilde{x}(s, t)=x(s,-t) \text { for } 0 \leq s<S,-T \leq t<0, \\
& \tilde{x}(s, t)=x(-s,-t) \text { for }-S \leq s<0,-T \leq t<0 .
\end{aligned}
$$

The two-variable function $\tilde{x}$ is symmetric with respect to the first argument and symmetric with respect to the second argument within the rectangle $[-S, S] \times[-T, T] ; \tilde{x}$ is $(2 S, 2 T)$-periodic in the whole plane $\mathbb{R} \times \mathbb{R}$.

Remark 3. For the $(2 S, 2 T)$-periodic function $\tilde{x}$, the four types of its Fourier coefficients in Definition 2 are reduced to a single type due to the symmetries of $\tilde{x}$ in both variables.

(1) The Fourier coefficients $B, C, D$ 's of $\tilde{x}$ are all zero.

$$
\begin{aligned}
& B_{m, n}(\tilde{x})=\frac{1}{S T} \int_{-S}^{S} \int_{-T}^{T} \tilde{x}(s, t) \sin \frac{m \pi s}{S} \cos \frac{n \pi t}{T} d s d t=0 \\
& C_{m, n}(\tilde{x})=\frac{1}{S T} \int_{-S}^{S} \int_{-T}^{T} \tilde{x}(s, t) \cos \frac{m \pi s}{S} \sin \frac{n \pi t}{T} d s d t=0 \\
& D_{m, n}(\tilde{x})=\frac{1}{S T} \int_{-S}^{S} \int_{-T}^{T} \tilde{x}(s, t) \sin \frac{m \pi s}{S} \sin \frac{n \pi t}{T} d s d t=0
\end{aligned}
$$

(2) For $x \in C[R]$, the function $\tilde{x}$ is therefore represented by a cosine $\times$ cosine series $A_{m, n}$ only:

$$
\tilde{x}(s, t)=\sum_{m, n=0}^{\infty}\left\{A_{m, n}(\tilde{x}) \cos \frac{m \pi s}{S} \cos \frac{n \pi t}{T}\right\}
$$

(3) By Parseval's identity,

$$
\sum_{m, n=0}^{\infty} A_{m, n}(\tilde{x})^{2}=\frac{1}{S T} \int_{-S}^{S} \int_{-T}^{T} \tilde{x}(s, t)^{2} d s d t
$$

Definition 4. We define spaces of double sequences.

(a) For $1 \leq p<\infty$, let $\ell^{p}=\left\{\left\{a_{m, n}\right\} \mid\left(\sum_{m, n=0}^{\infty}\left|a_{m, n}\right|^{p}\right)^{1 / p}<\infty\right\}$. 
(b) Let $\mathcal{H}$ be the space of all double sequences $\left\{a_{m, n}\right\}$ in $\ell^{1}$ with an inner product

$\left\langle\left\{a_{m, n}\right\},\left\{b_{m, n}\right\}\right\rangle=\sum_{k=0}^{\infty}\left(\sum_{l=0}^{k}\left(\frac{k(k+1)}{2}+l+1\right)^{2} a_{l, k-l} b_{l, k-l}\right)$.

For $\left\{a_{m, n}\right\}$ in $\mathcal{H}$, we let $\left\|\left\{a_{m, n}\right\}\right\|_{\mathcal{H}}=\sqrt{\left\langle\left\{a_{m, n}\right\},\left\{a_{m, n}\right\}\right\rangle}$.

(c) Let $\mathcal{U}$ be the space of all double sequences $\left\{a_{m, n}\right\}$ in $\ell^{2}$ such that the limit

$\lim _{p \rightarrow \infty} \frac{1}{\frac{p(p-1)}{2}+q+1}\left(\sum_{m=0}^{p-1} \sum_{u=0}^{m} a_{u, m-u} \cos \frac{u \pi s}{S} \cos \frac{(m-u) \pi t}{T}+\sum_{u=0}^{q} a_{u, p-u} \cos \frac{u \pi s}{S} \cos \frac{(p-u) \pi t}{T}\right)$ converges uniformly on $\mathbb{R} \times \mathbb{R}$. Here, $q$ is an integer and $0 \leq q \leq p$.

Remark 4. (a) For $1 \leq p \leq q$, $\ell^{p} \subset \ell^{q}$ holds.

(b) Throughout the paper, $\sum_{m, n=0}^{\infty} a_{m, n}$ represents a double sum. A double sum may not be equivalent to iterated sums. As known well, if a double series converges absolutely, then the double sum and iterated sums exist and are all equivalent. We will consider sequences in $\ell^{1}$ for our topic of the paper and the order of summation by rearrangement does not affect the convergence of such double series.

(c) The double sum $\sum_{m, n=0}^{\infty} a_{m, n}=\sum_{k=0}^{\infty} \sum_{l=0}^{k} a_{l, k-l}$ is carried out by diagonals, $a_{0,0}+\left(a_{0,1}+\right.$ $\left.a_{1,0}\right)+\left(a_{0,2}+a_{1,1}+a_{2,0}\right)+\cdots$, and such double series can be dealt with a single series. Furthermore, the method of summation by diagonals is known to be a practical method for the summation of double series.

(d) The space $\mathcal{U}$ is defined by associating with a double Fourier series; the limit of the arithmetic mean of partial sum sequences, say $\left\{S_{N}\right\}$ of the series, called a Cesàro mean or Cesàro summation. Let us assume that each term $a_{m, n}$ of the double series is a bead and we place the beads at the position $(m, n)$ in the first quadrant of the plane, starting from the first bead at the position $(0,0)$, the second bead at $(0,1)$, the third bead at $(1,0)$, and so on. With $S_{N}$, a set of of beads for a certain number $N$, we may fill up to the $(p-1)$-th diagonal and not all the $p$-th diagonal. The number of beads placed up to the $(p-1)$-th diagonal is $p(p-1) / 2$, from the expression $\sum_{m=0}^{p-1} \sum_{u=0}^{m} a_{u, m-u} \cos (\cdot) \cos (\cdot)$, and the number of the rest beads is $q+1$, from the expression $\sum_{u=0}^{q} a_{u, p-u} \cos (\cdot) \cos (\cdot)$. Obviously, $0 \leq q \leq p$, and $q=p$ when the $p$-th diagonal is filled. Therefore, the total number of terms involved in $S_{N}$ is $\frac{p(p-1)}{2}+q+1$. This value is reflected as a weight in the denominator within the limit of the space $\mathcal{U}$. This also motivates the definition of the space $\mathcal{H}$.

Theorem 2. $\mathcal{H} \subsetneq \ell^{1}$, that is, $\mathcal{H}$ is a proper subset of $\ell^{1}$.

Proof. Let $\left\{a_{m, n}\right\}$ be in $\mathcal{H}$. We manipulate its double sum in diagonal directions of indices; using Schwarz inequality twice,

$$
\begin{aligned}
\sum_{m, n=0}^{\infty}\left|a_{m, n}\right| & =\sum_{k=0}^{\infty} \sum_{l=0}^{k}\left|a_{l, k-l}\right|=\sum_{k=0}^{\infty} \sum_{l=0}^{k}\left\{\left(\frac{1}{\frac{k(k-1)}{2}+l+1}\right)\left(\left(\frac{k(k-1)}{2}+l+1\right)\left|a_{l, k-l}\right|\right)\right\} \\
& \leq \sqrt{\sum_{k=0}^{\infty} \sum_{l=0}^{k}\left(\frac{1}{\frac{k(k-1)}{2}+l+1}\right)^{2} \sqrt{\sum_{k=0}^{\infty} \sum_{l=0}^{k}\left(\left(\frac{k(k-1)}{2}+l+1\right)\left|a_{l, k-l}\right|\right)^{2}}} \\
& =\sqrt{\sum_{n=1}^{\infty} \frac{1}{n^{2}}}\left\|\left\{a_{m, n}\right\}\right\|_{\mathcal{H}}=\frac{\pi}{\sqrt{6}}\left\|\left\{a_{m, n}\right\}\right\|_{\mathcal{H}}<\infty,
\end{aligned}
$$

which implies that $\mathcal{H} \subset \ell^{1}$. In order to show that the two sets are unequal, we give an example sequence defined by $a_{m, n}=\frac{1}{\left\{\frac{(m+n)(m+n-1)}{2}+m+1\right\}^{3 / 2}}$. The norms of this sequence for each space are $\left\|\left\{a_{m, n}\right\}\right\|_{\ell^{1}}=\sum_{m, n=0}^{\infty}\left|a_{m, n}\right|=\sum_{k=1}^{\infty} \frac{1}{k^{3 / 2}}<\infty$, and $\left\|\left\{a_{m, n}\right\}\right\|_{\mathcal{H}}=\sqrt{\sum_{k=1}^{\infty} \frac{1}{k}}=$ $\infty$; this $\left\{a_{m, n}\right\}$ is a member of $\ell^{1}$ but not in $\mathcal{H}$. Therefore, $\mathcal{H} \varsubsetneqq \ell^{1}$. 
Lemma 1. $\left\{a_{m, n}\right\}$ is in $\ell^{1}$. Then, the series

$$
\sum_{m, n=0}^{\infty} a_{m, n} \cos \frac{m \pi s}{S} \cos \frac{n \pi t}{T}
$$

converges uniformly on $[-S, S] \times[-T, T]$.

Proof. Let $\epsilon$ be a given positive real number. As $\sum_{m, n=0}^{\infty} a_{m, n}$ converges, there are natural numbers $n^{*}, m^{*}$ such that $\sum_{m=m^{*}}^{\infty} \sum_{n=n^{*}}^{\infty}\left|a_{m, n}\right|<\epsilon / 3$. For $k=0,1, \ldots, m^{*}-1$, there is a natural number $n_{k}$ such that $\sum_{n=n_{k}}^{\infty}\left|a_{k, n}\right|<\frac{\epsilon}{3\left(n^{*}+1\right)}$ because $\sum_{n=0}^{\infty}\left|a_{k, n}\right|$ converges. Similarly, for $l=0,1, \ldots, n^{*}-1$, there is a natural number $m_{l}$ such that $\sum_{m=m_{l}}^{\infty}\left|a_{m, l}\right|<$ $\frac{\epsilon}{3\left(m^{*}+1\right)}$ because $\sum_{m=0}^{\infty}\left|a_{m, l}\right|$ converges. As usual, these $\epsilon-N$ methods are manipulated together by putting $u=\max \left\{m^{*}+n^{*}, n_{0}, n_{k}+1, \ldots, n_{k}+k, m_{l}, m_{l}+1, \ldots, m_{l}+l\right\}$. For $m+n \geq u$,

$$
\sum_{m+n \geq u}\left|a_{m, n}\right| \leq \sum_{m=0}^{m^{*}} \sum_{n=n_{m}}^{\infty}\left|a_{m, n}\right|+\sum_{n=0}^{n^{*}} \sum_{m=m_{n}}^{\infty}\left|a_{m, n}\right|+\sum_{m=m^{*}}^{\infty} \sum_{n=n^{*}}^{\infty}\left|a_{m, n}\right|<\frac{\epsilon}{3}+\frac{\epsilon}{3}+\frac{\epsilon}{3}=\epsilon .
$$

Then, by Weierstrass M-test,

$$
\sum_{m, n=0}^{\infty} a_{m, n} \cos \frac{m \pi s}{S} \cos \frac{n \pi t}{T}=\sum_{u=0}^{\infty}\left\{\sum_{m+n=u} a_{m, n} \cos \frac{k \pi s}{S} \cos \frac{(m-k) \pi t}{T}\right\}
$$

converges uniformly on $[-S, S] \times[-T, T]$.

Theorem 3. $\ell^{1} \varsubsetneqq \mathcal{U}$, that is, $\ell^{1}$ is a proper subset of $\mathcal{U}$.

Proof. Let $\left\{a_{m, n}\right\}$ be in $\ell^{1}$. Then, the series $\sum_{m, n=0}^{\infty} a_{m, n} \cos \frac{m \pi s}{S} \cos \frac{n \pi t}{T}$ converges uniformly by Lemma 1. Define

$$
\tilde{x}(s, t):=\sum_{m, n=0}^{\infty} a_{m, n} \cos \frac{m \pi s}{S} \cos \frac{n \pi t}{T} .
$$

on $[-S, S] \times[-T, T]$, then $\tilde{x}$ is continuous and symmetric with respect to both arguments. In fact, the series is the double Fourier series of $\tilde{x}$. Now we will prove that the limit (called Cesàro summation of Fourier series of $\tilde{x}$ ), denote it by $\beta$ for simplicity,

$$
\beta:=\lim _{p \rightarrow \infty} \frac{1}{\frac{p(p-1)}{2}+q+1}\left(\sum_{m=0}^{p-1} \sum_{u=0}^{m} a_{u, m-u} \cos \frac{u \pi s}{S} \cos \frac{(m-u) \pi t}{T}+\sum_{u=0}^{q} a_{u, p-u} \cos \frac{u \pi s}{S} \cos \frac{(p-u) \pi t}{T}\right)
$$

converges uniformly to $\tilde{x}$ on $[-S, S] \times[-T, T]$ for $0 \leq q \leq p$. For convenience, we denote $A_{m, n}(s, t)=a_{m, n} \cos \frac{m \pi s}{s} \cos \frac{n \pi t}{T}$ and $E(p, m, n, q)=\left\{\left(p^{\prime}, m^{\prime}, n^{\prime}, q^{\prime}\right) \mid m^{\prime}+n^{\prime}=\right.$ $p^{\prime}$, either $0 \leq p^{\prime}<p$ or $\left[p=p^{\prime}\right.$ and $\left.\left.0 \leq q^{\prime} \leq q\right]\right\}$ for all non-negative integers $p, m, n, q, p^{\prime}$, $m^{\prime}, n^{\prime}$ and $0 \leq q^{\prime} \leq q$. (here, $A_{m, n}(s, t)$ is used only within this proof and not the same as in Definition 2).

For convenience, we use a slightly different representation of the double sum, using triple summation. Let $\epsilon>0$ be given. Then, there are natural numbers $p^{*}$ and $q^{*}$ such that

$$
\left|\tilde{x}(s, t)-\sum_{m=0}^{p} \sum_{u=0}^{m} \sum_{k=0}^{u} A_{k, u-k}(s, t)\right|=\left|\tilde{x}(s, t)-\sum_{\left(p^{\prime}, m^{\prime}, n^{\prime}, q^{\prime}\right) \in E(p, m, n, q)} A_{k, u-k}(s, t)\right|<\epsilon
$$


for $p>p^{*}$ and $q>q^{*}$ for all $s, t$. The summation in Equation (2) can be grouped into two summations below. For $p>p^{*}$ and $q>q^{*}$,

$$
\begin{aligned}
& \sum_{\left(p^{\prime}, m^{\prime}, n^{\prime}, q^{\prime}\right) \in E(p, m, n, q)} A_{k, u-k}(s, t) \\
& =\sum_{\left(p^{\prime}, m^{\prime}, n^{\prime}, q^{\prime}\right) \in E\left(p^{*}, m, n, q^{*}\right)} A_{k, u-k}(s, t)+\sum_{m=p^{*}}^{p} \sum_{u=0}^{m} \sum_{k=q^{*}+1}^{q} A_{k, u-k}(s, t) \\
& \geq \sum_{\left(p^{\prime}, m^{\prime}, n^{\prime}, q^{\prime}\right) \in E\left(p^{*}, m, n, q^{*}\right)} A_{k, u-k}(s, t)+\left(\frac{p(p-1)}{2}-\frac{p^{*}\left(p^{*}-1\right)}{2}\right)(\tilde{x}(s, t)-\epsilon) .
\end{aligned}
$$

Here, the first term of a finite sum is of a finite value independent of $p$. Then,

$$
\frac{\sum_{\left(p^{\prime}, m^{\prime}, n^{\prime}, q^{\prime}\right) \in E(p, m, n, q)} A_{k, u-k}(s, t)}{\frac{p(p-1)}{2}} \geq \tilde{x}(s, t)-\epsilon \text { as } p \text { goes to infinity. }
$$

Similarly,

$$
\begin{aligned}
& \sum_{\left(p^{\prime}, m^{\prime}, n^{\prime}, q^{\prime}\right) \in E(p, m, n, q)} A_{k, u-k}(s, t) \\
& \leq \sum_{\left(p^{\prime}, m^{\prime}, n^{\prime}, q^{\prime}\right) \in E\left(p^{*}, m, n, q^{*}\right)} A_{k, u-k}(s, t)+\left(\frac{p(p-1)}{2}-\frac{p^{*}\left(p^{*}-1\right)}{2}\right)(\tilde{x}(s, t)+\epsilon) . \\
& \text { Then } \frac{\sum_{\left(p^{\prime}, m^{\prime}, n^{\prime}, q^{\prime}\right) \in E(p, m, n, q)} A_{k, u-k}(s, t)}{\frac{p(p-1)}{2}} \leq \tilde{x}(s, t)+\epsilon \text { as } p \text { goes to infinity. }
\end{aligned}
$$

Combining Equations (3) and (4), for an arbitrary positive real number $\epsilon$, we have

$$
\tilde{x}(s, t)-\epsilon \leq \beta \leq \tilde{x}(s, t)+\epsilon
$$

and the limit exists for all $(s, t)$. This implies that $\left\{a_{m, n}\right\}$ is in $\mathcal{U}$ and so $\ell^{1} \subset \mathcal{U}$.

In order to show that the two spaces are unequal, we give an example of an even continuous function whose Fourier series diverges at the origin. Let the function $g(x)=$ $\frac{\sin x^{2}}{x}$ for $x \neq 0$ on $[0, S]$ and $g(0)=0$. Then, $g$ is continuous at 0 . We define our twovariable function $f$ to be $f(x, y):=g(x)$ and even for both variables on $[-S, S] \times[-T, T]$. Then, $V_{1}(f)=\sup _{x}\left|f\left(x, y_{k}\right)-f\left(x, y_{k-1}\right)\right|=0, V_{3}(f)=\sup _{k} \mid f\left(x_{k}, y_{k}\right)-f\left(x_{k-1}, y_{k}-\right.$ $f\left(x_{k}, y_{k-1}+f\left(x_{k-1}, y_{k-1}\right) \mid=0\right.$, but $V_{2}(f)=\sup _{y}\left|f\left(x_{k}, y\right)-f\left(x_{k-1}, y\right)\right|=\infty$ Therefore, $\ell^{1}$ is a proper subset of $\mathcal{U}$.

Theorem 4. $\mathcal{U} \varsubsetneqq \ell^{2}$, that is, $\mathcal{U}$ is a proper subset of $\ell^{2}$.

Proof. We have $\mathcal{U} \subset \ell^{2}$ obviously from their definitions; it remains to show that $\mathcal{U} \neq \ell^{2}$. Assume that a sequence

$$
\left\{\frac{1}{\frac{p(p-1)}{2}+q+1}\left(\sum_{m=0}^{p-1} \sum_{u=0}^{m} a_{u, m-u} \cos \frac{u \pi s}{S} \cos \frac{(m-u) \pi t}{T}+\sum_{u=0}^{q} a_{u, p-u} \cos \frac{u \pi s}{S} \cos \frac{(p-u) \pi t}{T}\right)\right\}_{q=0, p=1}^{p, \infty}
$$

converges uniformly. At the point $s=0, t=0$, the sequence of real numbers $\left\{\frac{1}{\frac{p(p-1)}{2}+q+1}\left(\sum_{m=0}^{p-1} \sum_{u=0}^{m} a_{u, m-u}+\sum_{u=0}^{q} a_{u, p-u}\right)\right\}$ must converge as well. If we put $a_{m, n}=\frac{1}{\frac{(m+n)(m+n-1)}{2}+n+1}$, then $\sum_{m, n=0}^{\infty} a_{m, n}=\infty$ and $\sum_{m, n=0}^{\infty} a_{m, n}^{2}=\frac{\pi^{2}}{6}$, that is, $\left\{a_{m, n}\right\}$ is in $\ell^{2}$ 
but not in $\ell^{1}$. Furthermore, from the definition of a definite integral and Stirling's formula $\left(\sqrt{2 \pi} p^{p+1 / 2} e^{-p} \leq p ! \leq e p^{p+1 / 2} e^{-p}\right)$, we obtain

$$
\frac{\sum_{k=1}^{p}\left(\sum_{l=1}^{k} \frac{1}{l}\right)}{p} \geq \frac{\sum_{k=1}^{p} \int_{1}^{k+1} \frac{1}{x} d x}{p}=\frac{\ln ((p+1) !)}{p} \geq \frac{\ln \sqrt{2 \pi}+\left(p+\frac{3}{2}\right) \ln (p-1)-p}{p} \longrightarrow \infty
$$

as $p$ goes to infinity. This contradicts our assumption in Equation (5), which means that this $\left\{a_{m, n}\right\}$ is an $\ell^{2}$-member but not in $\mathcal{U}$.

Through the three theorems in this section, we examined the four spaces of double sequences, $\mathcal{H}, \mathcal{U}, \ell^{1}$, and $\ell^{2}$. We summarize the relationship of these four spaces as

$$
\mathcal{H} \varsubsetneqq \ell^{1} \varsubsetneqq \mathcal{U} \varsubsetneqq \ell^{2}
$$

This relationship of inclusion is explored to be used in the next sections.

\section{An Isomorphism between Double Fourier Coefficients and Two-Parameter Wiener Space}

Recall that $\mathcal{U}$ is the subspace of $\ell^{2}$ and defined by associating with double Fourier series. In this section, we examine an isomorphism $L^{2}(R) \rightarrow \ell^{2}$ who maps the twoparameter Wiener space to $\mathcal{U}$; for a member $x$ of the two-parameter Wiener space, a double Fourier series is associated with it and the limit of the arithmetic means of partial sums of the double Fourier coefficients converges uniformly.

The following notations are copied from Section 2 for the use in this section.

$R=[0, S] \times[0, T]$ is a rectangle in $\mathbb{R} \times \mathbb{R} ;$

$L^{2}(R)$ is the set of all square-integrable functions $f$ with respect to the Lebesgue measure;

The interested reader can find introductory background of the two-parameter Wiener space $C_{0}(R)$ in the author's recent work $[9,10]$.

Theorem 5. Let $J: L^{2}(R) \rightarrow \ell^{2}$ be a function with $J(x):=\left\{A_{m, n}(\tilde{x})\right\}$, where

$$
A_{m, n}(\tilde{x})=\frac{1}{S T} \int_{-S}^{S} \int_{-T}^{T} \tilde{x}(s, t) \cos \frac{m \pi s}{S} \cos \frac{n \pi t}{T} d m_{L}(s) d m_{L}(t)
$$

and $m_{L}$ is the Lebesgue measure on $\mathbb{R}$. Then, $J$ is an isometric isomorphism and $J\left(C_{0}(R)\right)=\mathcal{U}$.

Proof. By the uniqueness theorem for Fourier series of an $L^{2}$ function, $J$ is injective. From Parzeval's identity, $J$ is isometric. By Theorem $1, J\left(C_{0}(R)\right) \subset \mathcal{U}$. Then, it is sufficient to show that $\mathcal{U} \subset J\left(\mathcal{C}_{0}(R)\right)$. Let $\left\{a_{m, n}\right\}$ be in $\mathcal{U}$. Define

$$
\tilde{x}(s, t)=\lim _{p \rightarrow \infty} \frac{1}{\frac{p(p-1)}{2}+q+1}\left(\sum_{m=0}^{p-1} \sum_{u=0}^{m} a_{u, m-u} \cos \frac{u \pi s}{S} \cos \frac{(m-u) \pi t}{T}+\sum_{u=0}^{q} a_{u, p-u} \cos \frac{u \pi s}{S} \cos \frac{(p-u) \pi t}{T}\right)
$$

As $\left\{a_{m, n}\right\} \in \mathcal{U}$, the limit (Cesàro mean) implies uniform convergence with respect to $s, t$. Then, 


$$
\begin{aligned}
A_{v, w}(\tilde{x}) & =\frac{1}{S T} \int_{-S}^{S} \int_{-T}^{T} \tilde{x}(s, t) \cos \frac{v \pi s}{S} \cos \frac{w \pi t}{T} d s d t \\
& =\lim _{\substack{p \rightarrow \infty \\
1 \leq q \leq p}} \frac{1}{\frac{p(p-1)}{2}+q+1}\left(\sum_{m=0}^{p-1} \sum_{u=0}^{m} a_{u, m-u} \frac{1}{S T} \int_{-S}^{S} \int_{-T}^{T} \cos \frac{u \pi s}{S} \cos \frac{(m-u) \pi t}{T} \cos \frac{v \pi s}{S} \cos \frac{w \pi t}{T} d s d t\right. \\
& \left.+\sum_{u=0}^{q} a_{u, p-u} \frac{1}{S T} \int_{-S}^{S} \int_{-T}^{T} \cos \frac{u \pi s}{S} \cos \frac{(p-u) \pi t}{T} \cos \frac{v \pi s}{S} \cos \frac{w \pi t}{T} d s d t\right) \\
& =\frac{1}{S T} \lim _{\substack{p \rightarrow \infty \\
1 \leq q \leq p, v+w<p}} \frac{S T}{\frac{p(p-1)}{2}+q+1}\left(\frac{p(p-1)}{2}+q-\frac{(v+w)(v+w-1)}{2}-v\right) a_{v, w} \\
& =a_{v, w}
\end{aligned}
$$

As $\left\{A_{v, w}(\tilde{x})\right\}=J(x),\left\{a_{m, n}\right\}$ is in $J\left(C_{0}(R)\right)$, and this completes our proof.

There are interesting properties of our sequence spaces in relation to the isomorphism $J$.

Corollary 1. In relation to the isomorphism $\mathrm{J}$ in Theorem 5 , we have the following properties.

(a) Let $i_{1}:\left(C(R),\|\cdot\|_{\infty}\right) \rightarrow\left(L^{2}(R),\|\cdot\|_{2}\right)$ be an inclusion map. Then $i_{1}$ is injective continuous. As $C(R)$ is dense in $L^{2}(R),\left(J \circ i_{1}\right)(C(R))=\mathcal{U}$ is dense in $\ell^{2}$.

(b) Let $\left\{a_{m, n}\right\}$ be in $\ell^{2}$. For non-negative integers $p, q$, define $b_{m, n}^{p, q}=\left\{\begin{array}{ll}a_{m, n} & (\text { either } m<p \text { or } n<q) \\ 0 & \text { (otherwise) }\end{array}\right.$. Then, $b_{m, n}^{p, q}$ is in $\mathcal{H}$ and $b_{m, n}^{p, q}$ converges to $a_{m, n}$ in the sense of $\ell^{2}$-norm. Therefore, $\overline{\mathcal{H}}^{\|\cdot\|_{2}}=\overline{\mathcal{U}}^{\|\cdot\|_{2}}=\ell^{2}$.

We further study some subspaces of $\mathcal{U}, \mathcal{H}, \ell^{1}$, and $\ell^{2}$ defined in Section 3. Consider the Fourier series of a zero function. Let $x \in C_{2}(R)$ then $x(0,0)=0, x(s, 0)=0$, and $x(0, t)=0$ for all $s, t$. The Fourier series at the origin is $x(0,0)=\sum_{m, n=0}^{\infty} a_{m, n}$. By the uniqueness theorem of Fourier series, we have $\sum_{n=0}^{\infty} a_{m, n}=0, \sum_{m=0}^{\infty} a_{m, n}=0$ for each $m, n$ because $x(0,0)=0$. The Fourier series at $(0, t)$ is $x(0, t)=\sum_{n=0}^{\infty}\left(\sum_{m=0}^{\infty} a_{m, n}(x)\right) \cos \frac{n \pi t}{T}$. Again by the uniqueness theorem, $\sum_{n=0}^{\infty}\left(\sum_{m=0}^{\infty} a_{m, n}(x)\right)=0$ for each $n=0,1,2, \ldots$ because $x(0, t)=0$. Similarly, as $0=x(s, 0)=\sum_{m=0}^{\infty}\left(\sum_{n=0}^{\infty} a_{m, n}(x)\right) \cos \frac{n \pi s}{s}, \sum_{m=0}^{\infty}\left(\sum_{n=0}^{\infty} a_{m, n}(x)\right)=$ 0 for each $m=0,1,2, \ldots$ Being motivated by those series, we give the following subspaces.

Definition 5. Define the following subspaces of $\mathcal{U}, \mathcal{H}, \ell^{1}$, and $\ell^{2}$, respectively.

$$
\begin{aligned}
& \mathcal{H}_{0}=\left\{\left\{a_{m, n}\right\} \in \mathcal{H} \mid \sum_{n=0}^{\infty} a_{m, n}=0, \sum_{m=0}^{\infty} a_{m, n}=0 \text { for each } m, n\right\}, \\
& \ell_{0}^{1}=\left\{\left\{a_{m, n}\right\} \in \ell^{1} \mid \sum_{n=0}^{\infty} a_{m, n}=0, \sum_{m=0}^{\infty} a_{m, n}=0 \text { for each } m, n\right\}, \\
& \mathcal{U}_{0}=\left\{\left\{a_{m, n}\right\} \in \mathcal{U} \mid \sum_{n=0}^{\infty} a_{m, n}=0, \sum_{m=0}^{\infty} a_{m, n}=0 \text { for each } m, n\right\}, \\
& \ell_{0}^{2}=\left\{\left\{a_{m, n}\right\} \in \ell^{2} \mid \sum_{n=0}^{\infty} a_{m, n}=0, \sum_{m=0}^{\infty} a_{m, n}=0 \text { for each } m, n\right\} .
\end{aligned}
$$

Theorem 6. For the spaces $\mathcal{H}_{0}, \mathcal{U}_{0}, \ell_{0}^{1}$, and $\ell_{0}^{2}$, we have the following inclusion relationships,

$$
\mathcal{H}_{0} \varsubsetneqq \ell_{0}^{1} \varsubsetneqq \mathcal{U}_{0} \varsubsetneqq \ell_{0}^{2} ;
$$

and they are all Banach spaces.

Proof. We can obtain Equation (7) by the same methods as in the proof of Theorems 2-4 for the Formula (6) and thus do not write it here. We provide the proof of the second statement of Banach spaces. 
Let $\phi_{2, m}: \ell^{1} \rightarrow \mathbb{R}$ be a function defined by $\phi_{2, m}\left(\left\{a_{m, n}\right\}\right)=\sum_{n=0}^{\infty} a_{m, n}$ for each $m$. Then $\phi_{2, m}$ is linear. As

$$
\frac{\left|\phi_{2, m}\left(\left\{a_{m, n}\right\}\right)\right|}{\left\|\left\{a_{m, n}\right\}\right\|_{\ell^{1}}}=\frac{\left|\sum_{n=0}^{\infty} a_{m, n}\right|}{\sum_{m, n=0}^{\infty}\left|a_{m, n}\right|} \leq 1
$$

for $\left\{a_{m, n}\right\} \neq\{0\}$, the norm $\left\|\phi_{2, m}\right\|=1$. So $\phi_{2, m}$ is continuous. Therefore, the kernel of the function $\phi_{2, m}$, denoted by $\phi_{2, m}^{-1}(0)$, is a Banach space associated with $\ell^{1}$-norm; completeness comes from that the inverse image of a closed set $\{0\}$ of a continuous function is closed.

(ii) Let $\phi_{3, n}: \ell^{1} \rightarrow \mathbb{R}$ be a function defined by $\phi_{3, n}\left(\left\{a_{m, n}\right\}\right)=\sum_{m=0}^{\infty} a_{m, n}$ for each $n$. Then, $\phi_{3, n}$ is linear. As $\frac{\left|\phi_{3, m}\left(\left\{a_{m, n}\right\}\right)\right|}{\left\|\left\{a_{m, n}\right\}\right\|_{\ell 1}}=\frac{\left|\sum_{n=0}^{\infty} a_{m, n}\right|}{\sum_{m, n=0}^{\infty}\left|a_{m, n}\right|} \leq 1$ for $\left\{a_{m, n}\right\} \neq\{0\}$, the norm $\left\|\phi_{3, n}\right\|=1$. Thus, $\phi_{3, n}$ is continuous. Therefore, the kernel of $\phi_{3, n}, \phi_{3, n}^{-1}(0)$, is a Banach space associated with $\ell^{1}$-norm.

(iii) In fact, $\mathcal{U}_{0}=\left(\cap_{m=0}^{\infty} \phi_{2, m}^{-1}(0)\right) \cap\left(\cap_{n=0}^{\infty} \phi_{3, n}^{-1}(0)\right)$ and $\mathcal{U}_{0}$ is a Banach space.

(iv) Using a similar manner to these arguments, we can show that $\mathcal{H}_{0}, \ell_{0}^{1}$ and $\ell_{0}^{2}$ are also Banach spaces.

In fact, all of the four spaces- $\mathcal{U}, \mathcal{H}, \ell^{1}$, and $\ell^{2}$-are Banach spaces by the same method as Theorem 6 .

\section{Abstract Wiener Space of the Hilbert Space $\mathcal{H}$}

In this section, we prove that our Hilbert space of double sequences is an abstract Wiener space. Equipped with concepts in previous sections we are ready to state the concluding statement of this paper.

We here introduce the definitions and properties of an abstract Wiener space, mainly from in [4], necessary for our theorem so that one can follow this section without further references on an abstract Wiener space. We copy some of notations in Section 2 below, which can be easily found on functional analysis books.

- $\quad H$ be a real separable Hilbert space with norm $\|\cdot\|=\sqrt{\langle\cdot, \cdot\rangle}$.

- $\quad P$ is an orthogonal projection on $H$

- $\mathcal{F}$ is the partially ordered set of orthogonal projections $P$ of $H(P>Q$ means $P(H) \supset$ $Q(H)$ for $P, Q \in \mathcal{F})$

Definition 6 ([4], p. 3). A linear operator A of a separable Hilbert space $H$ is called a HilbertSchmidt operator if, for some orthonormal basis $\left\{e_{n}\right\}$ of $H, \sum_{n=1}^{\infty}\left\|A e_{n}\right\|^{2}<\infty$. The HilbertSchmidt norm of $A$ is defined as $\|A\|_{H}=\left(\sum_{n=1}^{\infty}\left\|A e_{n}\right\|^{2}\right)^{1 / 2}$.

Definition 7 ([4], p. 59). A semi-norm $\|\cdot\|$ in a Hilbert space $H$ is called measurable if for $\epsilon>0$, there exists $P_{0} \in \mathcal{F}$ such that $\mu\{\|P x\|>\epsilon\}<\epsilon$ for all $P \perp P_{0}, P \in \mathcal{F}$, where $\mu(E)=\left(\frac{1}{\sqrt{2 \pi}}\right)^{n} \int_{F} e^{-|x|^{2} / 2} d x$ for $E=\{x \in H: P x \in F\}$ and $n=\operatorname{dim}(P H)$.

Proposition 2 ([4], p. 59). Let $A$ be a Hilbert-Schmidt operator. Define $|x|=\|A x\|, x \in H$. Then,$|\cdot|$ is a measurable semi-norm.

The proposition has been complemented in [11], p. 276: A norm $|\cdot|$ is measurable iff there exists a one-to-one Hilbert-Schmidt operator $A$ such that $|x|=\|A x\|$.

Definition 8 ([4], p. 63). Let $B$ be the completion of $H$ with respect to a measurable norm $\|\cdot\| . i$ will denote the inclusion map of $H$ into $B$. The triple $(i, H, B)$ is called an abstract Wiener space.

We apply Proposition 2 to the space of sequences $\mathcal{H}$, and obtain the following theorem. 
Theorem 7. The norm $\|\cdot\|_{2}$ is a measurable norm on $\mathcal{H}$.

Proof. Let $T: \mathcal{H} \rightarrow \mathcal{H}$ be an operator defined by

$$
T\left(\left\{c_{m, n}\right\}\right):=\left\{\frac{c_{m, n}}{\frac{(m+n)(m+n-1)}{2}+n+1}\right\},\left\{c_{m, n}\right\} \in \mathcal{H}
$$

which is a double sequence, too. For non-negative integers $m, n$, define

$$
a_{m, n}(u, v):= \begin{cases}\frac{1}{\frac{(m+n)(m+n-1)}{2}+n+1} & \text { if }(u, v)=(m, n) \\ 0 & \text { otherwise }\end{cases}
$$

Then, $\left\{a_{m, n}\right\}$ is an orthonormal basis on $\mathcal{H}$, as for any $\left\{c_{m, n}\right\}, T\left(\left\{c_{m, n}\right\}\right)$ is actually an inner product with $\left\{a_{m, n}\right\}$. Then, for this sequence $\left\{a_{m, n}\right\}$,

$$
\left\|T\left(\left\{a_{m, n}\right\}\right)\right\|_{\mathcal{H}}^{2}=\sum_{n=0}^{\infty} \frac{1}{(n+1)^{2}}=\frac{\pi^{2}}{6} .
$$

As it is finite, $T$ is a Hilbert-Schmidt operator (see Definition 6). The Hilbert-Schmidt norm does not depend on the choice of an orthonormal basis of $\mathcal{H}$. For $\left\{c_{m, n}\right\}$ in $\ell^{2}$,

$$
\left\|\left\{c_{m, n}\right\}\right\|_{2}^{2}=\sum_{m, n=0}^{\infty} c_{m, n}^{2}=\left\|T\left(\left\{c_{m, n}\right\}\right)\right\|_{\mathcal{H}^{\prime}}^{2}
$$

that is, $\|\cdot\|_{2}$ is a measurable norm on $\mathcal{H}$ by Proposition 2.

Theorem 7 enables us to obtain the concluding statement of this paper.

Corollary 2. $\left(i, \mathcal{H}, \ell^{2}\right)$ and $\left(i, \mathcal{H}_{0}, \ell_{0}^{2}\right)$ are abstract Wiener spaces.

Proof. By Corollary 1 (b), $\ell^{2}$ is the completion of $\mathcal{H}$ with respect to the $\|\cdot\|_{2}$-norm. It is a measurable norm on $\mathcal{H}$ by Theorem 7 . With an inclusion map $i: \mathcal{H} \rightarrow \ell^{2}$, we conclude that $\left(i, \mathcal{H}, \ell^{2}\right)$ is an abstract Wiener space. These arguments are applied to the subspace $\mathcal{H}_{0}$ and $\left(i, \mathcal{H}_{0}, \ell_{0}^{2}\right)$ is also an abstract Wiener space.

For those who are interested in an analog Wiener space [6], the abstract Wiener space $\left(i, \mathcal{H}, \ell^{2}\right)$ is close to the sense of the analogue Wiener space; $\left(i, \mathcal{H}_{0}, \ell_{0}^{2}\right)$ is close to the sense of the two-parameter Wiener space, a classical Wiener sense.

\section{Conclusions and Discussion}

We explored four spaces of double sequences and developed them to establish abstract Wiener spaces. We created the space $\mathcal{U}$ of sequences of Cesàro means of double Fourier coefficients associated with two-variable functions. For the spaces of $p$-summable sequences of double sequences for $p=1,2, \ell^{1}$, and $\ell^{2}$, we used the same notations as that of single-indexed sequences. The Hilbert space $\mathcal{H}$ of double sequences is equipped with a Hilbert-Schmidt norm. We verified the inclusion relationships between these four spaces; they are nested as proper subsets.

We continued to pursue and prove that an isomorphic relationship mapped from the space of square-integrable functions to the space of 2-power summable sequences. By the isomorphism the two-parameter Wiener space $C_{0}(R)$ is isomorphic to $\mathcal{U}$, which played a key role for our last theorem. The completions of $\mathcal{H}$ and $\mathcal{U}$ were found to be identical and equivalent to $\ell^{2}$. Furthermore, all these properties are developed to our concluding statement of an abstract Wiener space; the Hilbert space $\mathcal{H}$ is an abstract Wiener space with an inclusion map to $\ell^{2}$. 
We think that our intermediate results of four spaces in Sections 3 and 4 are also significant, and they can provide a basis for the structure of the double sequence spaces. We expect them to be developed further as in the spaces of single-indexed sequences.

Funding: This research was supported by 2018 Hongik University internal Fund.

Acknowledgments: The author would like to thank K.S. Ryu at Hanam University, Korea for giving the motivation of this work.

Conflicts of Interest: The author declares no conflict of interest. The funders had no role in the design of the study; in the collection, analyses, or interpretation of data; in the writing of the manuscript; or in the decision to publish the results.

\section{References}

1. Weinberger, H.H.F. A First Course in Partial Differential Equations: With Complex Variables and Transform Methods; Dover Publications INC.: New York, NY, USA, 1965; Chapter 6, pp. 141-145.

2. $\mathrm{Wu}, \mathrm{W}$. Fourier Series and Fejér's Theorem. 2004. Available online: https://www.ocf.berkeley.edu/ wwu/articles / fejer_theorem. pdf (accessed on 8 June 2020).

3. Fejér, L. Sur les fonctions intégrables et bornées. Comptes Rendus l'AcadéMie Sci. 1900, 10, 984-987.

4. Kuo, H.-H. Gaussian Measures in Banach Spaces; Lecture Notes in Mathematics vol. 463; Springer: Berlin/Heidelberg, Germany; New York, NY, USA, 1975.

5. Gross, L. Abstract Wiener Measure; Lecture Notes in Mathematics vol. 140; Springer: Berlin, Germany, 1970.

6. Ryu, K.S. Analogue of Wiener integral in the space of sequences of real numbers. JCMS 2012, 25, 65-72. [CrossRef]

7. de Andrade, A.; Ruffino, P.R.C. Wiener integral in the space of sequences of real numbers. Arch. Math. 2000, 36, 95-101.

8. Poularikas, A.D. Fourier series. In The Handbook of Formulas and Tables for Signal Processing; Poularikas, A.D., Raton, B., Eds.; CRC Press LLC: New York, NY, USA, 1999.

9. Kim, J.-G. An Arc-Sine Law for Last Hitting Points in the Two-Parameter Wiener Space. Mathematics 2019, 7, 1131. [CrossRef]

10. Kim, J.-G. An average of surfaces as functions in the two-parameter Wiener space for a probabilistic 3D shape model. BKMS 2020, 57, 751-762. [CrossRef]

11. Tajkahashi, Y. On measurable norms and abstract Wiener spaces. Hokkaido Math. J. 1977, 6, 276-283. [CrossRef] 